\title{
Preventing the impact of selfish behavior under MANET using Neighbor Credit Value based AODV routing algorithm
}

\author{
K RAMA ABIRAMI $^{1, *(D)}$ and M G SUMITHRA ${ }^{2}$ \\ ${ }^{1}$ Department of Computer Science and Engineering, PSNA College of Engineering and Technology, \\ Dindigul 624308, India \\ ${ }^{2}$ Department of Electronics and Communication Engineering, Bannari Amman Institute of Technology, \\ Erode 638401, India \\ e-mail: ramaabirami1988@gmail.com;mg.sumithra1973@ieee.org
}

MS received 20 June 2016; revised 18 September 2017; accepted 22 November 2017; published online 13 April 2018

\begin{abstract}
Mobile Ad hoc Network (MANET) nodes exchange information using the multi-hop wireless communications without the need for any pre-existing infrastructure. Routing protocols of MANET are designed with an assumption that the nodes will cooperate in routing process. To achieve high throughput and reliable communication, the nodes are expected to cooperate with each other. Routing protocol plays a crucial role in an effective communication between nodes and operates on the assumption that the nodes are fully cooperative. Due to the open structure and limited battery-based energy in MANET, some nodes may not cooperate correctly or behave maliciously and such kind of misbehavior impacts the fairness, reliability and efficiency in MANET. Previous work addressed the ways to overcome these kinds of node misbehaviors and attacks. Most of the existing works need time to analyse the neighbor traffic and decide whether a neighbor is behaving maliciously or not. Further, the existing credit-based detection mechanisms may mark a genuine idle node as a malicious node. This work addresses a simple Neighbor Credit Value based AODV (NCV-AODV) routing algorithm for the detection of selfish behavior which avoids such false detection. The proposed idea is implemented in Ad hoc On Demand Distance Vector (AODV) routing protocol and an extensive analysis on the performance of the proposed detection mechanism against the selfish behavior of some MANET nodes are conducted.
\end{abstract}

Keywords. MANET; selfish behavior; routing protocols; AODV and security.

\section{Introduction}

Security in MANET is a vital concern for the fundamental working of a network. Availability of network services, confidentiality and integrity of the data can be achieved only by guaranteeing the security issues have been met. MANET is often prone to security attacks due to its features like open medium, dynamic topology, lack of central monitoring and management, cooperative algorithms and ambiguous defense mechanism.

The MANETs work with a decentralized administration in which the nodes communicate with each other based on mutual trust. This phenomenon in addition to wireless links makes MANETs more susceptible to attacks. Certain nodes in the network do not cooperate in order to save battery power and hence they act selfish by not forwarding the packets to the nearby nodes. Sometimes the nodes exhibit a malicious behavior such as a black hole attack which affects the communication and routing process significantly

*For correspondence
[1]. This work addresses the performance of the proposed selfish behaviors detection mechanism.

\subsection{Selfish node behaviors}

Selfish nodes are intended to enjoy the maximum profits from the networks and also they conserve their own resources like battery life, bandwidth, etc. A selfish node either refuses to retransmit the packet or drops them [2]. The features of the selfish nodes are non-participation in routing, no transmission or reply to HELLO messages, intentional postponement of Route Request (RREQ) packets and data packet dropping. According to the routing, there are three types of selfish nodes. They are as follows:

- Selfish node type 1 (SN1): These nodes involve in the Dynamic Source Routing (DSR) route discovery and route maintenance phases, but deny to forward data packets.

- Selfish node type 2 (SN2): These nodes do take part in neither the Route Discovery phase, nor forwarding data 
packets. They utilize their energy for transmission of their own packet.

- Selfish node type 3 (SN3): Depending upon the energy level, these nodes behave or misbehave differently in the network. The nodes behave properly when the energy lies between full energy E and a threshold T1. The node will behave of type SN1 at the time when the energy level is between T1 and another threshold T2. Finally, for an energy level lower than T2, it behaves like a node of type T2. The relationship between $\mathrm{T} 1$, $\mathrm{T} 2$ and $\mathrm{E}$ is $\mathrm{T} 2<\mathrm{T} 1<\mathrm{E}$.

Selfish node behavior attacks are having different impacts on performance and one particular type namely, the fully selfish node cannot be detected. So in this work, the first two types of selfish attacks are simulated and the third one which is the combination of first two types. SN2 type nodes are ignored by the routing protocol since they do not threaten the normal operation, even though they may degrade the network connectivity. The SN1 and SN3 types are more dangerous to the routing protocol. These nodes maintain the flow of route discovery traffic but interrupt the data flow, making the routing protocol to restart the routediscovery process or to select an alternative route if one is available. The newly selected routes may still include some of these SN1 type nodes, and hence the new route will also fail. This process will continue until the source concludes that the data cannot be transferred.

In this work, SN1 type attack is prevented using the proposed algorithm. Several methods have been proposed to deal with the problem of selfish node behavior. These methods can be divided into two categories [3]: Reputation based system [3, 4] and Credit based system [5]. Reputation based system monitors other behaviors to detect misbehaving nodes. If a node acts as a selfish node and drops other node's packet, it will earn bad reputation and will be isolated by other nodes. Credit based system rewards a node with certain credit when it forwards the packet of other node. If a selfish node does not forward other nodes packet, it loses and finally it is left with inadequate or no credits to forward its own data. The proposed model is a refined version of the second method and it overcomes some of the weaknesses in Credit based method outlined in the literature.

\section{Related works}

Most of the works to mitigate selfish behavior can be classified into Incentive-Based Mechanism [6], ReputationBased Mechanism [7] and Game Based mechanism [8]. The basic idea of the credit based mechanism is 'serve and earn'. The following related works focus on this type of mechanism.

Token Based Umpiring Technique (TBUT) to detect and eliminate the selfish nodes effectively in MANET [9].
There are two mechanisms in this method namely: packet dropping and selfish node quarantining mechanism. The selfish node is traced and identified in packet dropping detection mechanism. After detection, the offending nodes are marked and eliminated from the network using selfish node quarantining mechanism.

A virtual currency technique called as Nuglets (also called as beans) is earned by forwarding the packets in the network [10]. The security of the currency requires a trusted hardware which is the drawback of this approach.

An incentive based system [11] named SPRITE, in which selfish nodes are encouraged to cooperate. In this system, a node reports to the Credit Clearance Service (CCS), in which the messages that are received/forwarded by uploading its receipts. Intermediate node earns credit when they forward message of other nodes. In addition to the availability of central authority, SPRITE assumes source routing, public key infrastructure and makes use of a digital signature for any single transaction. In the network architecture of SPRITE, CCS is assumed to be reachable through internet hence limiting its utility.

A wireless health monitoring system using incentive based route cooperation [12] uses two cooperation protocols namely, Continuous Value Cooperation Protocol (CVCP) and Discrete Value Cooperation Protocol (DVCP) for improving message delivery reliability. CVCP protocol checks the value offered and stored credits. Credits are based on the basis of priority of the message. DVCP is designed for smaller ad hoc networks and uses approximate values such as High (H), Medium (M) and Low (L) to symbolize the incentive.

An incentive scheme in which every node determines the price of providing service for other nodes (such as forwarding their packet) [13]. A decision about this price is made based on the available bandwidth and battery power and it is assumed that the network nodes declare corresponding prices honestly. A source node makes use of the transmission path with lowest cost.

The ad hoc-VCG scheme [14] is a credit-based model which deals with the cooperation problem and introduces a second-best sealed type of auction. It ensures that a packet gets routed along the most energy-efficient path. A pricing question arises regarding the amount of the payment to a node should ask to forward packets. Intermediate nodes declare their respective prices genuinely which is assured by VCG mechanism. The ad hoc-VCG is robust when only one cheating node exists. It might fail in the presence of collision of nodes who try to maximize their payments. A game theoretic setting was introduced in this system for the routing layer, in which the communication nodes are assumed to be selfish and the communication nodes need to declare their cost of energy in order to compute a costefficient communication path, thus resulting in excessive overhead. It requires complete knowledge of the network topology during the route discovery phase. Finally, it does 
not target on the actual payment delivery, but only on the estimation of the payments.

Barter-based cooperation which is neither a credit-based nor reputation-based method simulates cooperation in the context of delay-tolerant networks [15]. A delay-tolerant wireless mobile ad hoc network transfers the messages from the source to its destination by the intermediate nodes in a store-forward manner. This system uses game theoretic model for the cooperation of the nodes.

A credit-based Secure Incentive Protocol (SIP) for simulating the cooperation in packet forwarding for MANET was studied [16]. SIP is based on credit-based charging and rewarding scheme by which nodes in an ad hoc network are charged or rewarded for the service they receive or provide. In order to prevent "credit fraudulence", SIP is carefully designed to guarantee the correct functioning of charging and also to defend a wide range of attacks. It takes a source controlled and session-based approach to reduce the communication and computation overhead. The limitations in this scheme are that it applies only to unicast traffic and not flexible or adaptable to network dynamics.

A secure multilayer credit-based incentive [17] scheme is to stimulate bundle forwarding operation among Delay Tolerant Networks (DTN) nodes. The proposed scheme can be implemented in a fully distributed manner to thwart various attacks without relying on any tamper proof hardware.

The general, mathematical frameworks via stochastic difference equations are used to model the interaction of incentive mechanisms and various underlying protocols [18]. This system presented a credit evolution model to quantify the expected credit variation of each node in Wireless Mesh Network (WMN), and then used the norm of the expected credits variation to quantify the credit disparity. It also proposed the use of differentiated pricing and showed how it could achieve credit equality among nodes, resulting in a more robust network under different traffic loading. Protocol Independent Fairness Algorithm (PIFA) which is a credit-based, centralized node fairness algorithm for MANET [19]. In this, the nodes are encouraged to gather credits by forwarding other's packet and use those credits to generate and forward their own packets. This mechanism makes the nodes to work cooperatively and avoids selfish behavior. In this algorithm, the nodes which try to drop packets and cheat the network management by reporting false packet forwarding information can be isolated and blacklisted from the network.

Limitations of all these credit based mechanisms are: (i) a virtual bank is required to manage credits, (ii) when a node has enough credits to send its own data, it can decide not to cooperate anymore and starts dropping packets, (iii) routing overhead is high and hence the network is instable, (iv) securing messages containing credits is also an essential requirement so that the malicious node could not change credit value, (v) did not pay attention to the fairness issue in routing when some nodes do not get reward due to some reasons, like location. The proposed NCV-AODV mechanism will address all these issues and prevent the selfish behavior of the nodes in the network.

\section{Modeling the attack and detection mechanism in AODV}

\subsection{The proposed NCV-AODV}

The proposed NCV-AODV is implemented by modifying the existing AODV protocol. Each node in the network will maintain a Neighbor Credit Table; in which it will update the credit value of its neighbor over time. Whenever a data packet is sent from a neighbor or forwarded by a neighbor, the neighbor is assumed as a genuine node and its credit value will be increased in the table. The genuine nodes that may not participate in any communication 'by chance' may also get poor credit value. To avoid this, each node in the network will send some minimum dummy data to get credit in their neighbor credit table.

Whenever a node needs to resolve a route through a next hop node or forward a packet through a next hop node, it will check the credit value of that next hop node in its neighbor credit table and will only use that hop if that node has minimum credit value. Otherwise, it will just drop that packet. The following section shows the modification made in AODV to make it work as NCV-AODV.

\subsection{The proposed attacks detection mechanism}

The function AODV:: recv will be called on the event of receiving any packet at routing layer. The following pseudo code 1 explains the way in which the credit of a neighbor is updated on the event of receiving a data packet from it. If a node receives a packet from a previous hop neighbor, then the credits of previous hop node as well as the original source node of the packet were increased.

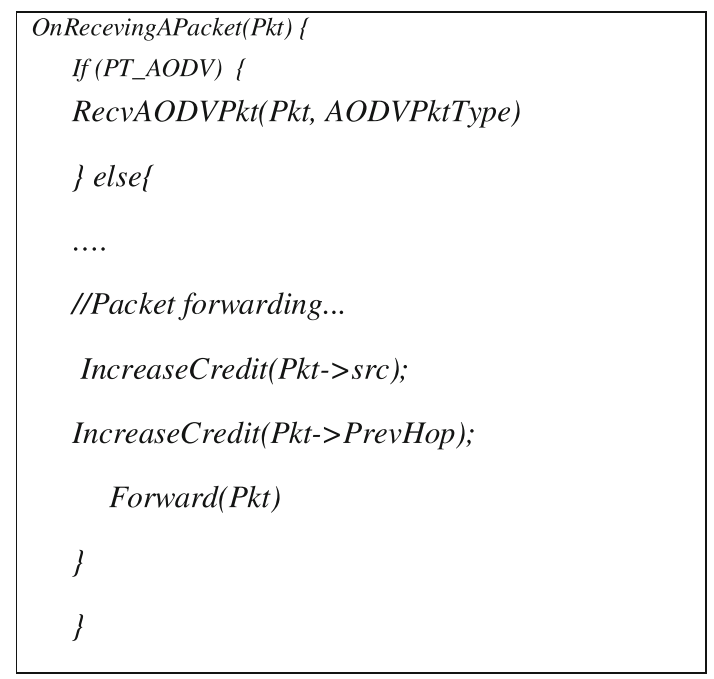

Pseudo Code 1. Explaining the Modifications in NCV-AODV:: recv 
The function rtable.rt_lookup will be called on the event of resolving a route to a destination at routing layer. The following pseudo code 2 explains the way in which the route resolving process is controlled with respect to the credit value of the next hop node. If a node tends to forward a packet to next hop which has a lesser credit value than a threshold value, then that route will get disabled. So that, next possible route will be resolved latter.

\begin{tabular}{|} 
OnRouteLookup (Dst) \{ \\
RT=FindRoute(Dst) \\
If(GetCredit $(\mathrm{RT}->$ NextHop $))<$ \\
CreditThreshold $)$ ( \\
Dissable $($ RT $) ;$ \\
Return $(0) ;$ \\
Return $(R T)$ \\
\}
\end{tabular}

Pseudo Code 2. Explaining the Modifications in NCVAODV_rtable::rt_lookup()

The following Pseudo Code 3 shows the timer function added in AODV to send dummy data in a very controlled fashion. The dummy data is transmitted as one hop broadcast at very long intervals to avoid flooding of the network and increase of the message overhead. So, every genuine node in the network will send at least few dummy data packets to its neighbors to get good credit value at its neighbors. This mechanism avoids the detection of genuine nodes as malicious nodes. Without this mechanism, the genuine nodes that will not send or receive any packets may get marked as malicious. The nodes that were actively sending packets will not trigger this timer function. So, this mechanism will work without any additional overhead.

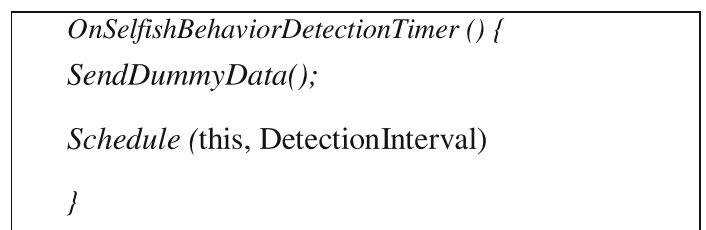

Pseudo Code 3. Shows the Timer Function Added in NCV-AODV

Most of the existing credit based mechanism will mark the nodes that were not sending any packet or not having a chance to forward any packet as malicious. Our proposed model will not mark such nodes in the network.

The function IncreaseCredit() is shown in the following Pseudo Code 4. This function will increase the credit value of the node.

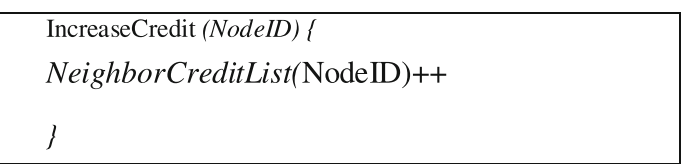

Pseudo Code 4. Shows IncreaseCredit() Function Added in NCV-AODV

The following pseudo code 5 explains the function SendDummyData(). Even though this function may be called periodically by a timer function or from any other function, it will not send dummy data during each and every call to this function. This function will actually send data, if and only if that node is idle for some time. This mechanism avoids the increase in overhead.

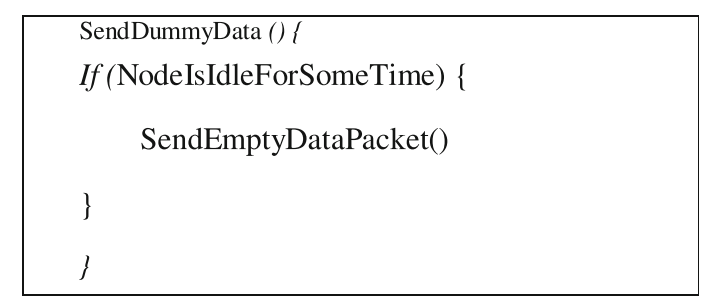

Pseudo Code 5. Shows SendDummyData() Function Added in NCV-AODV

The malicious behavior is avoided in two locations in the AODV code. The first one is at "route lookup" stage and the second one is at "on receiving an AODV packet". The following pseudo code 6 is the second location where the request from a potential malicious node is avoided. As shown in the pseudo code, if a packet is received from a lower credit neighbor, then it was just dropped without any further processing.

RecvAODVPkt (Pkt, AODVPktType) \{
If(Detection) \{
If (GetCredit(Pkt->PrevHop)
$<$ CreditThreshold)
//packet from a low credit node - so just drop it
Free(Pkt);
Return();
\}
switch(AODVPktType) \{
case TYPE_RREQ:
RecvRequest(Pkt)
break
case RREP:
RecvReply(Pkt);
break
case RERR:
RecvError (Pkt);
break
case HELLO:
\} //End of Switch
\}//End of RecvAODVPkt

Pseudo Code 6. Explaining the modifications in NCV-AODV:: recvAODV 
The following Pseudo Code 7 shows the function that returns the credit of a node.

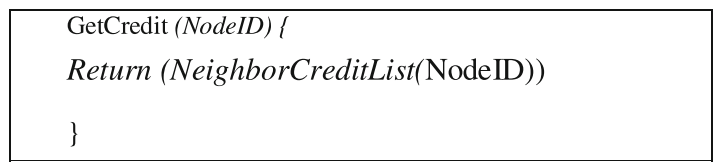

Pseudo Code 7.shows the return the credit of node

So, one AODV routing agent code will act as normal AODV or NCV-AODV or Malicious AODV with respect to the initialization parameter.

This approach is adapted since more than one routing agent cannot be used in a single node under NS2. So it is designed as a single routing agent that can work more than one way with respect to initialization parameters.

\section{Simulation parameters and evaluation metrics}

\subsection{About the simulator}

The paper uses the most popular and freely available simulator NS2 (Network Simulator version 2.35). NS is a discrete event simulator used for networking research. In the simulation, the wireless channel capacity of mobile hosts uses 2 Mbps with the propagation of two ray ground and a nominal range of 250 meters with an omni directional antenna. NS-2 implements the standard IEEE 802.11 Distributed Coordination Function (DCF) MAC protocol. Table 1 shows the parameters which are used in the simulation.

Table 1. Traffic parameters.

\begin{tabular}{lc}
\hline Topography-X size & $800 \mathrm{~m}$ \\
Topography-Y size & $800 \mathrm{~m}$ \\
No. of Background Traffic & 10 \\
$\quad$ Flows & \\
Background Traffic Type & CBR (Constant Bit Rate) \\
Transport Agent & UDP \\
CBR-packet size & $512 \mathrm{bytes}$ \\
CBR-interval & $1 \mathrm{~s}$ \\
CBR-rate & $10 \mathrm{~kb}$ \\
Traffic Start Time & 30 th sec \\
Traffic Stop Time & 100 th sec \\
Mobility Model & Random Way Point \\
Important Parameters & \\
NoMaliciousNodes & 5 \\
AODV States & Normal AODV \\
& AODV with Selfish Behavior \\
& TypeI \\
& NCV-AODV \\
\hline
\end{tabular}

\subsection{Metrics considered for evaluation}

The performance of the ad hoc routing protocols is evaluated using the following metrics:

- Maliciously Dropped Packet: This is an important metric which shows the number of packets maliciously dropped at routing layer due to attack.

- Throughput: Throughput is the number of bytes or bits arriving at the sink over time. It is generally measured in kilo bits per second or Mega bits per second.

- Packet Delivery Fraction/Ratio (PDF/PDR): $\sum$ Number of packet receive / $\sum$ Number of packet send

- End to End delay: Average time in order to traverse the packet inside the network.

- MAC Load: It is the ratio of the number of MAC layer messages generated by every node to the number of data packets delivered successfully to the entire destination node.

- Routing Load: Routing load is measured by the ratio of the number of routing messages generated (not forwarded) by the nodes in the network to the number of data packets successfully delivered to all the destination nodes.

\section{Results and discussion}

The results show the performance with respect to different number of nodes in the network. In this experiment, the number of malicious nodes is kept as 5 and the performance is compared with normal AODV without any malicious nodes.

Figure 1 shows the performance of the proposed detection mechanism in terms of maliciously dropped packets at routing layer. As shown in the following graph, the packet maliciously dropped at routing layer is very much controlled in the proposed detection model. This method makes the NCV-AODV to behave just equal to that of normal AODV without any attack.

Figure 2 shows the performance of the proposed detection mechanism in terms of maliciously dropped packets at application layer. The following graph clearly shows the maliciously dropped packets at application layer are very much controlled in the proposed detection model when compared with AODV under selfish type I attack and AODV without malicious nodes.

Figure 3 shows the performance of the proposed detection mechanism in terms of throughput. The following graph infers that the throughput is higher in detection (NCV-AODV) than normal AODV. So the proposed detection and prevention mechanism improved the throughput considerably.

Figure 4 shows the performance of proposed detection mechanism in terms of PDF. In the following graph, the detection NCV-AODV shows higher PDF when compared 


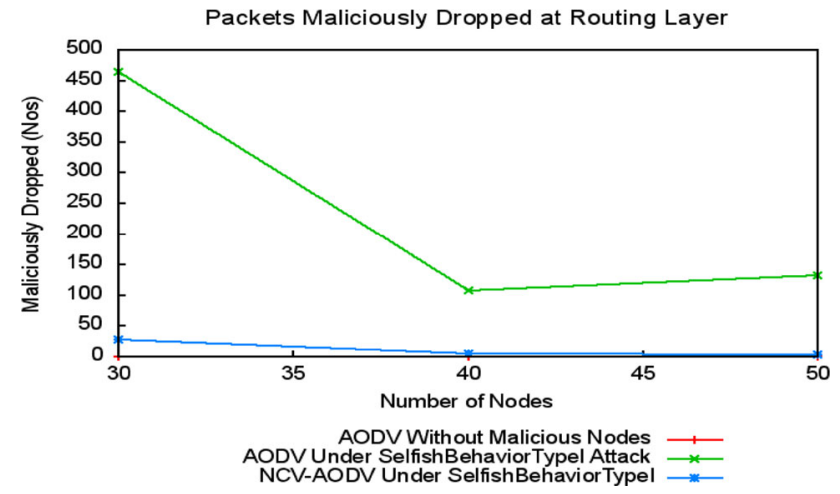

Figure 1. Number of nodes vs. maliciously dropped packets at routing layer.

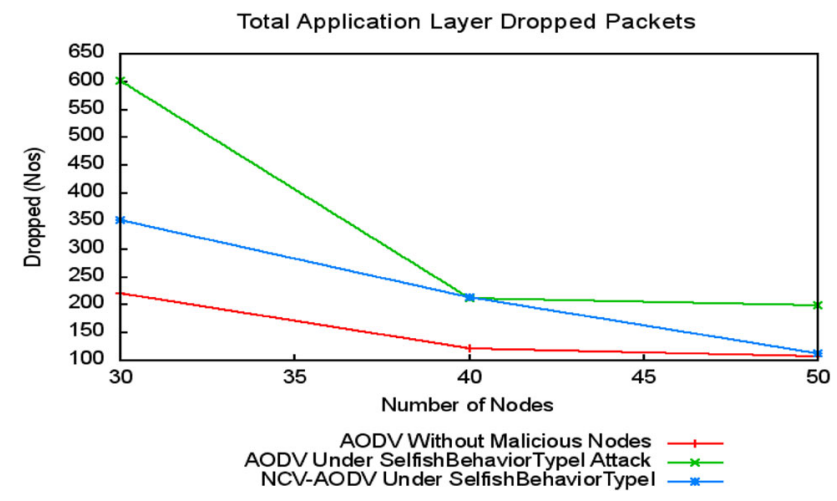

Figure 2. Number of nodes vs. dropped packets at application layer.

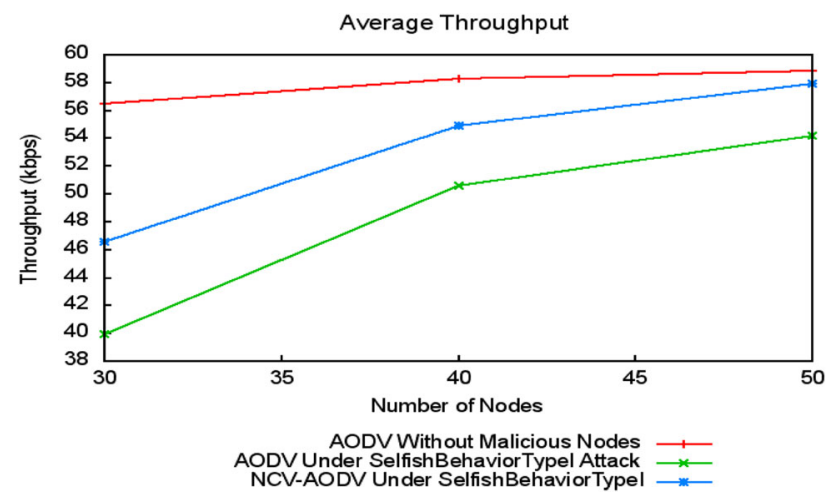

Figure 3. Number of nodes vs. throughput.

with AODV without detection. Hence the proposed detection and prevention mechanism improved the PDF considerably.

Figure 5. shows the performance of proposed detection mechanism in terms of end to end delay. It is inferred from the graph that the detection NCV-AODV shows low end to end delay than the AODV without detection.

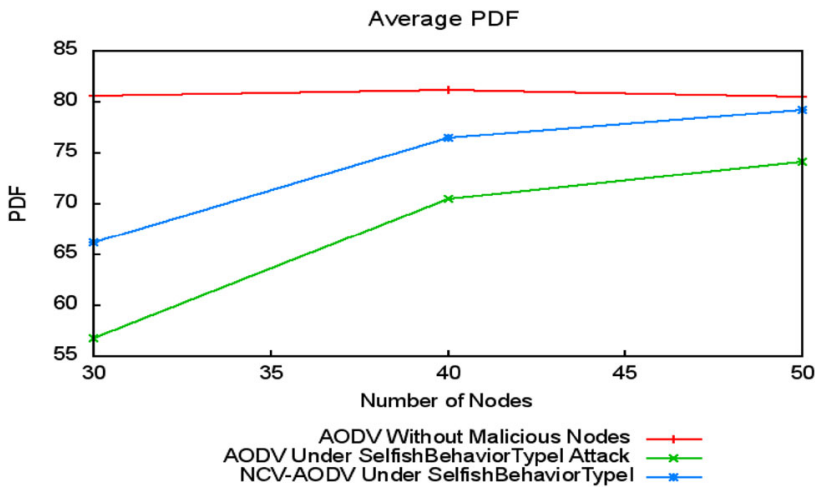

Figure 4. Number of nodes vs. PDF.

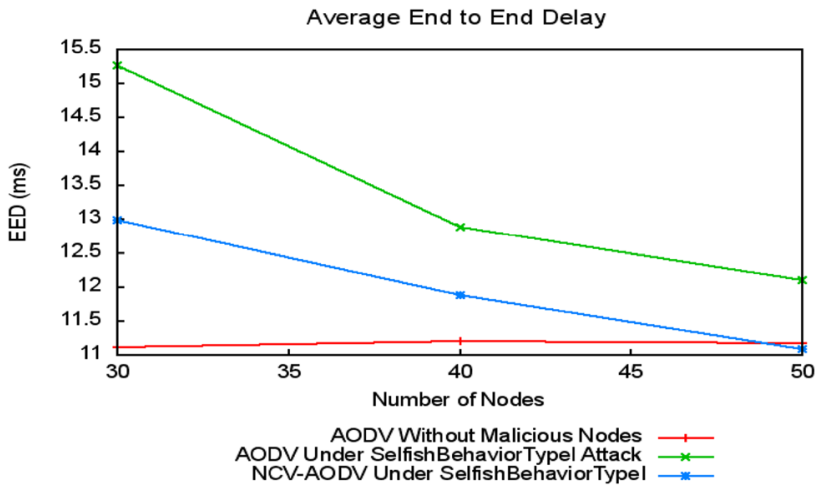

Figure 5. Number of nodes end to end delay.

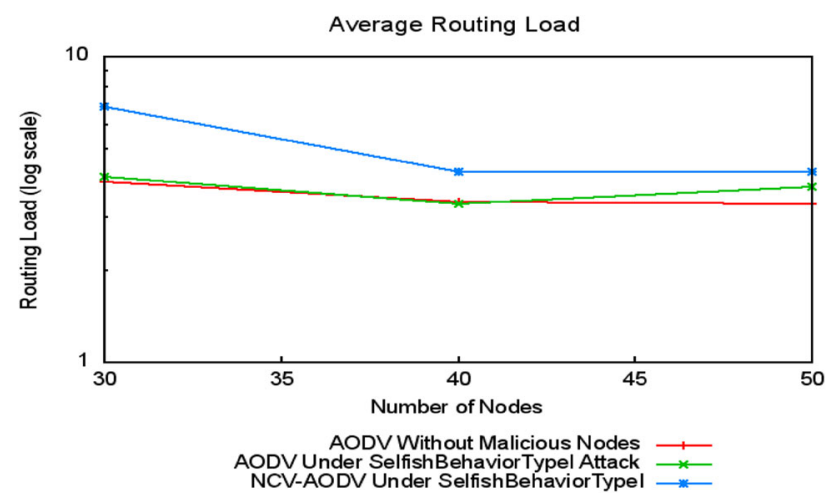

Figure 6. Number of nodes vs. routing load.

Figure 6 shows the performance of the proposed detection mechanism in terms of Routing Load. As shown in the following graph, NCV-AODV shows not much additional Routing Load when compared with AODV. The increase in Routing Load actually signifies the improvement in performance as it was able to send and receive more packets. So, here the increase in routing load shows the positive improvement. 


\section{Conclusion}

This work has addressed a simple and efficient way to prevent selfish behavior of nodes under MANET. The proposed mechanism will work better with respect to the increase of data transmission activity in the network. So, under a much active network, it will resolve the credit value of neighbors in an efficient way and provide better performance without any noticeable increase in detection overhead.

Most of the previous "credit based" detection methods will work by hearing or overhearing the neighbor data transmissions. So, if a neighboring node which is not actively sending or receiving any data may be misclassified as a malicious node. To overcome this problem, the proposed method will simulate some dummy data traffic from such idle genuine nodes to prevent them marked as malicious.

As shown in the results of the previous section, the proposed methods successfully detected the selfish neighbor nodes and avoid sending packets through them which obviously improved the overall performance.

\section{References}

[1] Praveen Joshi 2011 Security issues in routing protocols in MANETs at network layer. Procedia Computer Science. 3: 954-960

[2] Ryu B G, Choi J H and Lee S 2013 Impact of node distance on selfish replica allocation in a mobile ad-hoc network. $A d$ Hoc Network 11(8): 2187-2202

[3] Marti S, Giuli T, Lai K and Baker M 2000 Mitigating routing misbehavior in mobile ad hoc networks. Mobile Computing and Networking. 255-265

[4] Buchegger S and Boudec J Y L 2002 Performance Analysis of the CONFIDENT PROTOCOL: Coperation of NodesFairness in Distributed Ad-Hoc Networks. In: IEEE/ACM Workshop on Mobile Ad Hoc Networking and Computing(MobiHOC), Lausanne

[5] Buttayan L and Hubaux J P 2003 Stimulating Cooperation in Self-Organizing Mobile Ad Hoc Networks. ACM/Kluwer Mobile Networks and Applications, vol. 8, pp. 579-592

[6] Hales D 2004 From Selfish Nodes to Cooperative Networks Emergent Link-based Incentives in Peer-to-Peer Networks. In: IEEE International Conference on Peer-to-Peer Computing. pp. 151158
[7] Rebahi Y, Mujica V and Sisalem D 2005 A ReputationBased Trust Mechanism for Ad hoc Networks. In: 10th IEEE Symposium on Computers and Communications. pp. 37-42

[8] Mahajan R, Rodrig M, Wetherall D and Zahorjan J 2005 Sustaining cooperation in multi-hop wireless networks. NSDI.

[9] Jeba kumar M, Kathirvel A, Kirubakaran N, Sivaraman P and Subramaniam M 2015 A unified approach for detecting and eliminating selfish nodes in MANETs using TBUT. EURASIP Journal on Wireless Communications and Networking, vol. 2015, pp.143

[10] Buttayan L, and Hubaux J P 2000 Enforcing service availability in mobile ad-hoc WANs. In: Proceedings of IEEE/ ACM Workshop on Mobile Ad Hoc Networking and Computing MobiHoc. Boston

[11] Zhong S, Chen J and Yang Y R 2003 Sprite: A simple, cheat proof credit based system for mobile ad hoc networks. In: Proceedings of IEEE INFOCOM, San Francisco, CA, United States. pp. 1987-1997

[12] Varshney U 2008 Improving Wireless Health Monitoring Using incentive Based Router Cooperation. IEEE Computer Society. 41(5): 56-62

[13] Crocraft J, Gibbens R, Kelly F and Ostring S 2004 Modeling Incentives for collaboration in mobile ad hoc networks. Performance Evaluation 57(4): 427-439

[14] Anderegg L and Eidenbenz S 2003 Ad hoc-VCG: A truthful and cost-efficient routing protocol for mobile ad hoc networks with selfish agents. In: 9th International Conference Mobile Computing and Networking, San Diego, CA. pp. 245-259

[15] Buttyan L, Dora L, Félegyhazi M and Vajda I 2007 Barterbased cooperation in delay tolerant personal wireless networks. In: IEEE Workshop on Autonomic and Opportunistic Communications, AOC.

[16] Yanchao Z, Wenjing L, Wei L and Yuguang F 2007 A secure incentive protocol for mobile ad hoc networks. Wireless Networks (WINET) 13(5): 569-582

[17] Haojin Z, Xiaodong L, Rongxing L, Yanfei F and Xuemin 2009 SMART: A Secure Multilayer Credit-Based Incentive Scheme for Delay-Tolerant Networks. IEEE Transactions on Vehicular Technology 58(8): 4628-4639

[18] Honghuing L, Patrick P C, Lee, John C S and Lui 2013 On the credit evolution of credit-based incentive protocols in wireless mesh networks. Journal of Computer Networks. 57: 3327-3343

[19] Younghwan Y, Sanghyun A and Agarwal D P 2005 A creditpayment scheme for packet forwarding fairness in mobile ad hoc networks. In: IEEE International Conference on Communications. vol. no. 5, pp. 3005-3009 